\title{
eJRIEPS
}

Ejournal de la recherche sur l'intervention en éducation physique et sport

Hors-série $N^{\circ} 1 \mid 2015$

Quelques aspects théoriques de la didactique des sports collectifs. Préparation aux concours de recrutement

\section{L'opposition et ses concepts associés en sports collectifs}

Jean-Francis Gréhaigne

\section{OpenEdition}

Journals

Édition électronique

URL : http://journals.openedition.org/ejrieps/1308

DOI : $10.4000 /$ ejrieps. 1308

ISSN : 2105-0821

Éditeur

ELLIADD

Référence électronique

Jean-Francis Gréhaigne, "L'opposition et ses concepts associés en sports collectifs », eJRIEPS [En ligne], Hors-série $\mathrm{N}^{\circ} 1$ | 2015, mis en ligne le 01 décembre 2015, consulté le 03 octobre 2019. URL : http://journals.openedition.org/ejrieps/1308; DOI : 10.4000/ejrieps.1308

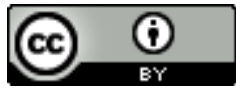

La revue eJRIEPS est mise à disposition selon les termes de la Creative Commons Attribution 4.0 International License. 
eJRIEPS Hors série n¹ Décembre 2015

L'opposition et ses concepts associés en sports collectifs

Jean-Francis Gréhaigne* \& Guillaume Dietsch ${ }^{\star *}$

* Professeur des Universités retraité, Université Bourgogne Franche-Comté

** Professeur Agrégé d'EPS, Lycée Professionnel Aristide Briand Le Blanc-Mesnil, Doctorant au LIRTES (EA7313), Université Paris-Est

De nombreuses recherches dans le passé et dans le présent se sont intéressées aux sports collectifs. Un sport collectif est un sport qui oppose des équipes entre elles, par opposition aux autres sports où l'on est tout seul. Cette notion d'opposition comprend les jeux en opposition indirecte (jeu en parallèle, jeu en opposition simultanée, jeu à camps différenciés) et les jeux d'opposition directe et interpénétrée avec des statuts différenciés des joueurs et des jeux avec statuts indifférenciés pour les joueurs. Par ailleurs, il existe beaucoup de classifications concernant les sports collectifs. Parmi les plus courantes, qui diffèrent selon les caractéristiques choisies, nous pouvons citer :

Sport collectif de grand terrain - sport collectif de petit terrain.

Sport collectif de préhension - sport collectif de frappe.

Sport collectif interpénétré - sport collectif non interpénétré...

Sport à cible verticale - Sport à cible horizontale - Sport à cible mixte (Gréhaigne, 1989).

Pourtant, il ne faut ne pas confondre sport collectif et sport d'équipes tels certains sports comme l'aviron (qui est un sport individuel en skiff et un sport d'équipe à partir du deux barrés) ou encore contre-la-montre par équipe en cyclisme. Autre exemple, en tennis, la Coupe Davis représente aussi une compétition à part. Elle rassemble des joueurs habitués à s'entraîner et à jouer seuls, parfois les uns contre les autres. Les sports en doublette comme au beach-volley ou le double dans les sports de raquettes qui reposent sur un couple de joueurs contre un autre couple, ne sont pas des sports collectifs. Déjà dans sa thèse en 1989, Gréhaigne affirmait qu'il n'y a pas de sports collectifs en dessous du trois contre trois sinon pour des situations infra sport collectif qui visent l'étude d'un cas particulier.

Dans les sports collectifs, les règles premières fondent la logique des jeux dans la prise en compte systématique du rapport d'opposition entre une attaque et une défense. Les conséquences de cette conception du jeu sont considérables. Comprendre ce rapport de forces est un passage obligé pour que tous les élèves investissent la singularité de 


\section{eJRIEPS Hors série n¹ Décembre 2015}

l'activité ou son essence. Pour définir l'essence c'est-à-dire le rapport fondamental qui fonde cette activité (Eloi \& Uhlrich, 2001), nous faisons le constat que, comme pour d'autres sports collectifs, ce jeu requiert deux cibles et un seul projectile (Deleplace, 1983). Le ressort du jeu est donc fondé sur le fait qu'il faut être en possession du ballon pour pouvoir tenter d'atteindre la cible adverse. Ainsi, ce dispositif engendre une véritable lutte entre protagonistes adverses pour entrer en possession du ballon. II s'agit de gérer simultanément d'un côté la conservation de la balle pour son équipe et la conquête de la cible opposée, ou bien de l'autre la conquête de la balle et la défense de sa propre cible. De plus, l'idée de groupes restreints qui s'affrontent (Mérand, 1977) et celle d'efficacité constituent bien l'essence des sports collectifs. Pour Conquet et Devaluez (1986), l'essence de l'activité est donc un concept qui renvoie au sens ; au pourquoi plutôt qu'au comment, au contenu plutôt qu'aux apparences, à la fonction plutôt qu'à la forme (p. 92). Pour compléter ce rapide tour d'horizon historique, citons Brackenrigde (1979) pour qui les sports collectifs sont une lutte pour la conquête d'un territoire avec un ensemble de règles qui incluent des stratégies, des tactiques significatives et des aspects techniques où l'anticipation / coïncidence est de la plus grande importance. La lutte pour la conquête d'un territoire est réglée par un système de score qui symbolise l'importance de la victoire. Le règlement identifie les problèmes et garantit pour l'ensemble des équipes ou des individus l'égalité des chances. Enfin, pour Mérand (1977, p. 12) «les jeux sportifs collectifs sont une modalité originale d'exploitation de la dynamique des groupes restreints poursuivant un objectif commun ». La dynamique des groupes qui s'opposent s'exprime dans la circularité des problèmes à poser et des problèmes à résoudre en vue d'atteindre à la fois l'objectif qui finalise l'action des joueurs partenaires, tout en faisant obstacle aux entreprises des joueurs adversaires. Les multiples possibilités de placement et de déplacements donnent au jeu un caractère d'incertitude, renforcé par le fait que l'initiative change de camp avec le gain ou la perte de la balle.

Le problème fondamental des sports collectifs pourrait être ainsi énoncé : "dans un rapport d'opposition, il s'agit de coordonner les actions collectives afin de récupérer, conserver, faire progresser le ballon dans le but d'amener celui-ci dans la zone de marque et de marquer. A cet effet, le plus souvent il faut prendre de l'avance sur le replacement défensif, conserver cette avance et réussir la réalisation. 》(Gréhaigne, 1989, p. 13). L'objet de chacune des actions offensives est bien de provoquer et d'exploiter un déséquilibre du dispositif adverse, de créer l'effet de surprise, bref l'imprévisible, afin de marquer un but ou un point. Les attaquants doivent s'efforcer de prendre de vitesse la 


\section{eJRIEPS Hors série n¹ Décembre 2015}

reconstitution par l'équipe opposée d'un équilibre défensif ou d'amener le barrage adverse dans une position critique et ainsi rompre l'équilibre à leur avantage (Mahlo, 1969 ; Marle, Pasteur \& Voland, 1996). Chaque joueur y bâtit du sens et progresse. L'essentiel des situations d'apprentissage s'envisage alors en opposition et les rapports d'opposition se trouvent au centre de la transformation des élèves.

Sans ce milieu didactique approprié où l'opposition est centrale pour toute analyse, on en revient à l'approche de la nage à sec. Or, dans l'apprentissage de la natation, on a depuis longtemps cessé de faire réaliser des mouvements sur un tabouret pour les refaire, ensuite, dans l'eau en faisant monter celle-ci progressivement. Pour en revenir au sport collectif, en procédant de la sorte, on met en place un système particulier de repérage, où les aspects tactiques des configurations du jeu et les informations provenant de la présence active de l'adversaire au lieu d'être le guide de l'action, deviennent littéralement perturbantes au lieu d'être guide de l'action.

Pour compléter ce premier tour d'horizon, nous allons envisager les apports de quelques concepts et autres notions dialectiques pour mieux appréhender les problèmes que posent les sports collectifs et qui ont des conséquences directes sur sa didactique.

\section{Opposition / coopération}

On parle d'opposition lorsque deux faits forment un contraste ou lorsque l'un fait obstacle à l'autre. Parfois, l'opposition est la relation de deux objets ou de deux groupes placés l'un en face de l'autre. Depuis l'antiquité, ce concept d'opposition est le plus souvent présenté sous deux formes fondamentales : la contradiction ou la contrariété. C'est l'un des acquis les plus anciens de la logique formelle mais aussi l'un des plus féconds, tant il permet d'analyser des phénomènes très divers qui traitent, du désordre. Au XVIIle siècle, la doctrine logique classique de ces deux types d'opposition a subi un remodelage, avec l'introduction du concept de dynamique, occasionnée par le développement de la physique et des sciences de la vie. Plus récemment, dans les années 1950-1970, avec le développement du structuralisme, le concept d'opposition a connu un grand regain d'intérêt, notamment avec pour ce qui nous concerne, les recherches de Claude LéviStrauss en anthropologie (Lévi-Strauss, 1955). En logique formelle, deux termes sont dits opposés quand ils sont contraires ou contradictoires : c'est bien le cas des deux équipes en sport collectif (Gréhaigne, 1992a).

Dans les sports collectifs, le renversement dans la conception du jeu a consisté à considérer que rien ne peut être expliqué et réalisé sans une bonne compréhension des 


\section{eJRIEPS Hors série n¹ Décembre 2015}

rapports d'opposition liant constamment les deux équipes au fil du déroulement du jeu. Dans ces conditions, l'intelligence tactique est donc l'art et la science de déterminer ce que l'opposition fait ou pourrait faire, pour empêcher l'accomplissement de la tâche. Elle est utilisée pour appuyer la prise de décisions relatives à la planification de l'affrontement et son exécution afin d'anticiper ce qui peut arriver dans la bataille. A ce propos, Carnot (1810), formule des principes d'action : primauté de l'offensive, actions par surprise, décisions rapides et actions de masse. II faut attaquer toujours l'ennemi là où il est faible et avec une supériorité de forces telle que la victoire ne puisse jamais être douteuse. L'auteur différencie stratégie et tactique : la tactique est l'art d'organiser et de disposer ses forces pour gagner une bataille ; la stratégie est l'art de mobiliser ses forces, d'organiser et de disposer ses batailles pour gagner la guerre.

Pour approfondir ces premiers éléments concernant tactique et stratégie nous allons nous appuyer sur les écrits de deux stratèges de la guerre renommés Sun Tzu et Von Clausewitz (Gréhaigne, 2014). Bien sûr les sports collectifs ne sont pas la guerre. II n'empêche que nous pouvons tirer des enseignements des données fournies par ces deux auteurs en les adaptant pour les jeux collectifs interpénétrés et de signes contraires (Jeu, 1977). Globalement le plan de jeu type «Von Clausewitz » vise à imposer son jeu et maîtriser totalement l'adversaire. II s'agit pour l'entraîneur de dresser une forme d'action idéale sous forme de schéma ou d'un plan de jeu contraignant. Ensuite, toutes les actions ou interventions consistent à appliquer les solutions retenues. Quant à un plan de jeu de type Sun Tzu, il consiste à laisser venir l'adversaire pour mieux le surprendre et économiser ensuite ses forces. Plutôt que de dresser un modèle qui serve de norme à son action, le ou les joueurs devraient concentrer leur attention sur le cours du jeu dans lequel ils se trouvent engagés pour en déceler la cohérence et profiler son évolution. II faut s'attacher à détecter les facteurs favorables dans la configuration du jeu. En un mot s'appuyer sur le potentiel de la situation (Jullien, 1992). Face à cette incertitude de l'évolution du jeu, les composantes fondamentales de toute prédiction restent le prévisible et l'aléatoire. L'aléatoire étant par définition imprévisible, dans ce contexte, les maîtres mots sont flexibilité et adaptabilité. La stratégie qui renvoie souvent à une forme de planification formelle, peut apparaître comme un facteur d'inertie. Cette vision n'est pas tout à fait juste si l'on envisage la planification comme étant de portée générale. Elle laisse ainsi suffisamment de souplesse à la tactique pour garantir l'efficacité face à l'opposition. Stratégie et réactivité sont de ce fait mieux associées permettant d'assurer une certaine autonomie aux différents niveaux d'organisation. Au final, l'opposition constitue une notion 


\section{eJRIEPS Hors série n¹ Décembre 2015}

centrale et avec Jeu (1977), nous ajouterons afin que la compétition ait un vrai sens pour le joueur qu'il faut respecter la maxime " de l'égalité des chances à l'inégalité du résultat " (p 131). En effet, cette caractéristique définit bien les préalables à l'affrontement et la finalité du jeu. Nous sommes assez loin du modèle coopératif où l'état d'esprit et le mode de comportement des joueurs reposent, avant tout, sur des relations et des échanges non conflictuels voire non concurrentiels. La mise en place de modalités appropriées pour analyser ensemble et de façon partagée les situations et collaborer dans le même esprit pour parvenir à des fins communes ne suffit pas en sport collectif pour gagner ou analyser le jeu en sport collectif car l'essence de l'activité n'y est pas.

Analyser et expliquer l'opposition et utiliser cette opposition comme source de tout progrès, tant en compréhension qu'en exécution permet de concevoir des situations à l'école ou à l'entraînement qui, tout en permettant de faire jouer la réalité de l'opposition, gardent une visée d'apprentissage. En se centrant sur des exercices avec opposition, on propose aux joueurs de les accoutumer à des configurations qu'ils retrouveront lors du match, mais aussi de les placer en crise temporelle ce qui nécessitera une adaptation rapide face au problème posé.

\section{Sens et opposition}

Cette notion de sens pourrait être définie de la façon suivante : c'est une représentation en partie liée à une appartenance sociale en rapport avec une conception de la logique de l'activité. En un mot, c'est ce qui organise la finalité des actions des élèves. Le sens renvoie à la formalisation implicite ou explicite que le sujet se donne des observations et des consignes de la tâche avant de les traiter. L'adaptation ou non de l'équipe et des joueurs aux conditions de l'affrontement permet de déterminer les statuts de dominant et de dominé. L'équipe dominante est celle qui attaque activement. II faut néanmoins bien différencier la possession du ballon de la notion de dominant / dominé car on peut très bien choisir "d'attaquer l'attaque " à certains endroits stratégiques du terrain. Pour l'instant, chez les élèves, on peut identifier les catégories suivantes.

- Les élèves qui rentrent directement dans la notion d'opposition et pour qui progresser veut dire quelque chose.

- Les élèves pour qui la coopération semble l'élément organisateur de leurs actions. Cela se traduit souvent par de la passe à 10 .

- Les élèves pour qui en dehors des règles fédérales et de la pratique sociale de référence, «c'est pas du foot». 


\section{eJRIEPS Hors série n¹ Décembre 2015}

- Les élèves qui avant tout veulent se faire plaisir, se détendre et «bien jouer » où le souci de progresser est second par rapport à la récréation. Ils ont simplement envie de faire « un p'tit match ».

- Les élèves qui calquent leur comportement sur les joueurs de haut niveau et cherchent à les imiter comme par exemple l'élève orienté par les gestes techniques qualifié de « joueur perso » par Caillé (2008).

Dans la lignée des analyses de Marcel Mauss, Caillé (2008) propose en effet une représentation de l'action humaine oscillant entre quatre pôles (comme les aiguilles d'une boussole) et traversée par deux axes (liberté / obligation - intérêt pour soi / intérêt pour autrui). Cette typologie s'applique au monde du football. En effet, le joueur doit se soumettre à des obligations (placement sur le terrain, efforts de repli défensif) et dispose aussi d'une importante liberté (dribbler ou donner la balle). Dans le même temps, il est à la fois soucieux de la réussite collective (gagner le match, combiner ses actions avec celles de ses partenaires) et de son intérêt individuel (faire la différence, réaliser de beaux gestes). Ces quatre dimensions fonctionnent de pair. Aucun joueur n'est totalement libre et égoïste, de même qu'aucun n'est rigidement contraint. Ce constat d'une certaine irréductibilité de ces quatre déterminants de l'action n'empêche pas de dégager des types-idéaux de joueurs, selon le poids relatif des déterminants dans leur conduite sur le terrain. Cette synthèse permet de réfléchir sur les caractéristiques des joueurs en prenant en compte le poids du contexte institutionnel, tout en laissant des marges à l'acteur. Quatre types de joueurs sont identifiés: le «versatile", le «rigoureux», le joueur «perso », le « créatif ».

Chez les filles, on peut repérer, quand elles jouent avec des garçons, des comportements particuliers. Ces conduites se retrouvent parfois chez les garçons lorsque le niveau des joueurs à l'intérieur d'une même équipe est par trop hétérogène :

. les «pom pom girls» qui stationnent au milieu du terrain et lèvent les bras quand les garçons marquent ;

- celles ou ceux qui participent au jeu mais qui restent centrés sur la coopération en rapport avec des problèmes de statut dans l'équipe ou de refus d'opposition.

D'autres comportements peuvent être repérés en particulier chez les élèves en difficulté :

- ceux qui ne font rien et parfois fuient le ballon «les nuls » ou débutants (ceux-là qu'on oublie souvent quand on se limite à la pratique de jeux réduits sans observation),

- ceux ou celles qui font du demi-fond ; 


\section{eJRIEPS Hors série n¹ Décembre 2015}

- ceux qui veulent bien coopérer et échanger à condition de gagner ;

- ceux ou celles qui se réfugient dans les apprentissages techniques... ou le fait de ne pas savoir (ceux-là qu'il faut amener vers la compréhension des situations d'opposition jouées).

II est à noter que ces comportements varient suivant les élèves, suivant les moments, suivant les facteurs extérieurs et suivant les sports collectifs. La notion de rôle, conduite de l'élève ou du joueur qui exprime ce qu'il pense avoir à faire en fonction de la façon dont il vit le rapport de forces, devient essentielle pour appréhender sa place dans le groupe. De plus, cette notion est aussi influencée par son rapport avec les autres dans l'équipe et comment il gère ses ressources dans ce système de contraintes.

\section{Equilibre / déséquilibre}

Dans le jeu, les réseaux de forces ne sont ni neutres ni homogènes. Le jeu est un champ de forces fait d'équilibres et de déséquilibres momentanés où la volonté d'aller de l'avant est contrebalancée par la crainte de perdre le contrôle du ballon. Cette tension est au cœur de la dynamique du jeu. Et plus il y a d'informations à traiter, plus il y a de déséquilibre potentiel. Effectivement, une perturbation provoque un éloignement de cet état d'équilibre. L'équipe doit alors développer des mécanismes qui tentent d'amortir cette perturbation pour revenir à une certaine stabilité. Avec ce mouvement, le système est confronté à la notion du temps et la notion de la phase, puisqu'il fonctionne, la plupart du temps, sur un régime stationnaire. Ces mécanismes peuvent conduire à des asymétries temporelles très prononcées, à des comportements spatio-temporels inattendus ou à l'apparition de comportements chaotiques. II faut savoir gérer cette tension entre l'ouverture qui conquiert et la fermeture qui protège. La valeur de l'information n'est plus liée à la quantité. Elle ne se capitalise plus. Elle acquiert une valeur dans l'échange et dans l'interaction du moment.

En effet, ce qui nous intéresse est plutôt relatif aux conditions permettant une description des propriétés régissant l'évolution du système vers des états d'équilibre ou de déséquilibre car ce dernier présente, sur le fond, un équilibre souvent d'une autre nature en fonction de la cinétique du jeu. Des états finis de configurations connues sont nécessaires pour agir par rapport à l'infini des configurations possibles. Dans cette perspective, le développement de la compréhension des états de non-équilibre a entraîné de nombreux perfectionnements. Si l'on considère que l'ordre est assimilable à l'équilibre, le progrès est de pouvoir passer de l'ordre vers le désordre ou l'inverse sans déstabiliser 


\section{eJRIEPS Hors série n¹ Décembre 2015}

complètement l'équipe. On peut également distinguer le déséquilibre créé dans le rapport d'opposition au regard de la configuration antérieure et l'équilibre dynamique recréé dans le mouvement offensif en cours, avec la recherche de réorganisation de l'offensive pour entretenir la stabilité du jeu.

Comme nous l'avons déjà évoqué, dans la pratique sociale de référence "football », le jeu en mouvement au milieu du terrain recèle la logique d'exploitation des situations de déséquilibre, qu'elle tend à faire s'enchaîner « en culbute " (Deleplace, 1994). Une telle filiation semble bien pouvoir être dégagée si, au lieu de rester prisonnier de l'ordre chronologique d'apparition des phases dans la séquence de jeu, on part du fait que la logique d'organisation de cette première action se retrouve dans toutes les autres, qui ne sont alors que des cas particuliers. Cette phase-mère du jeu constitue fréquemment une situation de rupture, le lieu d'apparition de déséquilibres à l'origine des attaques, un lieu où l'affrontement pour la récupération du ballon a été le plus fort. Dans les phases d'équilibre, l'étude de la phase-mère du jeu montre souvent une défense bien étagée sur le terrain en fonction du plan de jeu choisi. Dans ce cas, on parlera souvent de stabilité et "d'équilibre défensif » dans le sport collectif considéré. Cette notion est intéressante, mais il faut autant que possible la cerner avec précision si l'on veut s'en servir. L'équipe en train de défendre réalise l'« équilibre défensif » à un instant donné du mouvement de jeu quand, à cet instant, la distribution de l'ensemble de ses joueurs est telle qu'elle est en mesure de répondre sur le champ et efficacement à chacune des éventualités que l'équipe adverse peut tenter dans l'instant immédiatement suivant. Cela doit se réaliser et peu importe le rapport à l'adversaire, le rapport au ballon et le rapport au terrain. On sait que dans la réalité du jeu concret, il arrive qu'à un instant d'équilibre défensif succède un autre instant d'équilibre défensif. Mais il arrive aussi qu'à un instant d'équilibre défensif momentané suit un instant de déséquilibre, etc. C'est-à-dire qu'un déséquilibre peut aussi bien être la conséquence d'une mauvaise distribution l'instant d'avant que d'une mauvaise utilisation de la bonne distribution qui existait l'instant d'avant. Néanmoins, un déséquilibre défensif peut être récupéré par l'équilibre défensif étant reconstitué l'instant d'après.

Du point de vue de la continuité ou de la rupture à propos des configurations du jeu en cours, il est possible de caractériser les points de rupture. Quand cette rupture de l'état d'équilibre a déjà eu lieu, il est le plus fréquemment dû à une défaillance dans l'organisation défensive. On est face à un déséquilibre initial qui peut entraîner une défense à la «poursuite » ou une défense en "barrage » mais avec une grande infériorité numérique. Les transitions et le jeu de transition renvoient souvent à des configurations où 


\section{eJRIEPS Hors série n¹ Décembre 2015}

l'on a un peu de temps pour agir car la densité des joueurs y est souvent moins importante. L'attaque, alors, doit profiter du moment de déséquilibre pour conserver son avance éventuelle tandis que la défense doit rapidement revenir en barrage ou y rester. $\mathrm{Si}$, le déséquilibre n'existe pas, comment le faire apparaître ? Certes, un des bons moyens est de tenter d'amener le ballon en avant de l'espace de jeu effectif. Avec un équilibre défensif bien en place et stable, il faut s'en remettre à un exploit tactico / technique de l'attaquant ou à une erreur, une maladresse défensive qui est à exploiter très rapidement. Anticiper devient alors un élément clé de la réussite. L'anticipation, c'est l'action de prévoir ce qui va arriver à partir d'hypothèses ou de suppositions. C'est l'activité adaptatrice que développe le joueur par rapport à une future configuration du jeu, lorsqu'il suppose sa prochaine apparition. L'anticipation est essentielle à la réalisation de l'efficacité des projets de jeu et des tactiques individuelles propres à chaque sport collectif pour prévoir les aléas du jeu pour décider vite et juste. Pour cette raison, la compréhension des processus qui la constituent est essentielle pour l'enseignant et l'entraîneur. II leur faut être capable de concevoir et conduire un processus de perfectionnement permettant d'agir sur la prise de décision tactique, le tout combiné avec la préparation de l'équipe à la compétition (Deleplace, 1983).

Concernant le joueur, celui-ci doit concentrer son attention sur le cours du jeu, tel qu'il s'y trouve engagé, pour en déceler la cohérence et profiler son évolution, en bref s'appuyer sur le potentiel de la situation. On peut dire que deux notions se trouvent ainsi au cœur de l'analyse du jeu. D'une part, celle de configuration du jeu telle qu'elle s'actualise et prend forme dans le rapport de forces en course et d'autre part celle du potentiel de cette configuration du jeu.

\section{Ordre / désordre}

Dans une rencontre, l'opposition entre les équipes engendre de l'imprévu et la nécessité constante de s'adapter aux contraintes issues de l'affrontement. Un match n'est que très rarement la simple application de combinaisons tactiques apprises à l'entraînement. Ainsi dans le jeu, le plus souvent, on ne peut envisager, le plus fréquemment, que des probabilités d'évolution des configurations de l'attaque et de la défense, d'où l'importance d'heuristiques pour traiter plus vite les problèmes posés par l'interaction spécifique des deux équipes.

Ce type d'analyse qui privilégie l'opposition et la "gestion du désordre " comme source de perfectionnement semble mettre en évidence de nouveaux concepts dont la prise en 


\section{eJRIEPS Hors série n¹ Décembre 2015}

compte paraît fondamentale pour un enseignement rénové des sports collectifs. De plus, elle permet de réhabiliter le jeu comme élément fondamental de l'apprentissage et pour paraphraser un slogan célèbre qui voulait rendre l'eau aux piscines « restituons le champ de jeu aux footballeurs ".

La figure 1 décrit quelques concepts qui apparaissent dès que l'on met l'opposition comme facteur fondamental au centre du processus didactique en sport collectif. Un exemple d'analyse pourrait être le suivant. Le porteur de balle, qui n'est ni en avance ni en retard sur le jeu, transmet le ballon à une partenaire proche sans prise de risque pour assurer la conservation du ballon. Ceci garantit ainsi la stabilité des échanges car il ne recherche pas un déséquilibre immédiat.

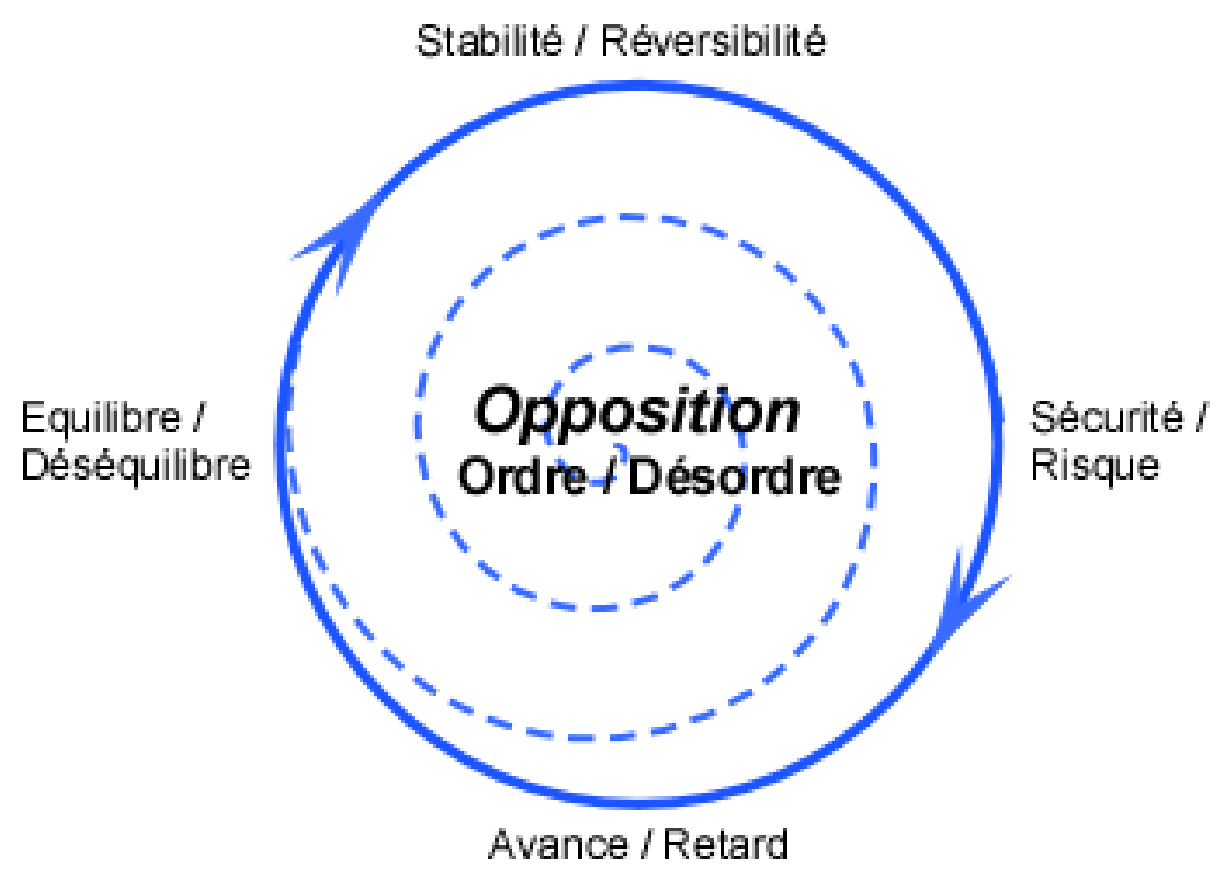

Figure 1. Description des concepts découlant de la notion d'opposition.

Cette notion centrale d'opposition nous a amené à considérer les deux équipes comme des systèmes organisés en interaction. Les caractéristiques structurelles de ces systèmes consistent en un programme, modifiable en fonction de l'expérience acquise. Leur principale propriété fonctionnelle est l'apprentissage.

Du point de vue de la didactique des sports collectifs, une conséquence importante pourrait découler de l'approche systémique que nous avons menée et ainsi constituer une nouvelle hypothèse de recherche didactique. Dans l'apprentissage classique des sports collectifs on cherche avant tout à enseigner aux élèves des gestes techniques et à imposer de l'ordre sur le terrain sous forme de répartition formelle par exemple. En tirant 


\section{eJRIEPS Hors série n¹ Décembre 2015}

les conséquences de notre travail, nous serions tenté de dire qu'il est aussi important, et peut-être même plus, d'amener les joueurs à gérer de façon optimale le désordre (Villepreux, 1987 ; Gréhaigne, 1989). En effet, dans une rencontre l'opposition génère de l'imprévu et une nécessité constante de s'adapter aux contraintes issues de l'affrontement. Une rencontre n'est que très rarement la simple application de schémas tactiques répétés sans fin à l'entraînement. Les attaquants sont amenés à gérer du désordre dans le jeu en mouvement, ainsi qu'à créer et/ou exploiter des déséquilibres fugaces dans le rapport de forces. Les conduites décisionnelles nécessitent alors la réduction de deux types d'incertitude : évènementielle et temporelle. «Avance / retard ", "barrage / poursuite " deviennent des notions importantes pour prendre des décisions. Enfin, nous sommes en accord avec Menaut (1982) quand il conclut en disant : " on ne saurait comprendre et expliquer la complexité de l'objet des jeux sportifs collectifs, en tant que système de transformation, qu'en faisant appel à un modèle intégrant les notions d'ordre, de désordre, d'interaction et d'organisation » ( $p$ 78).

\section{Stabilité / réversibilité}

Au plan général, la réversibilité opératoire selon Piaget (1974) constitue un mécanisme essentiel des opérations mentales, qui permet de réaliser des relations d'inversion et de réciprocité entre différents éléments. Ces éléments servent à résoudre les problèmes posés par les configurations du jeu. En sport collectif, la réversibilité souligne tout d'abord, l'immédiateté du passage d'attaquant à défenseur et met en évidence la notion de situation à double effet (Deleplace, 1979). La réversibilité des situations représente un aspect fondamental des sports collectifs en rapport avec le fait que les équipes attaquent ou défendent à tour de rôle. Cette réversibilité ou stabilité est à considérer dans un rapport dialectique continuité / rupture de l'action en cours de deux façons :

- soit grâce à une circulation du ballon et des joueurs permettant la mise en place d'une configuration opportune qui peut amener une rupture momentanée de l'état d'équilibre du système attaque / défense. Toutefois, cette action peut amener un danger de but si l'exécution est rapide et bien assurée. Également, il faut enchaîner vers d'autres configurations du jeu sans perdre le ballon pour attendre ou provoquer une autre opportunité pour assurer la continuité de l'action vers le but en cas d'échec;

- soit grâce à une rupture définitive de l'action de jeu par perte de la balle, les défenseurs récupérant la balle et deviennent attaquants. Des configurations du jeu seront alors plus adaptées que d'autres pour réussir un but en fonction du lieu de récupération. 


\section{eJRIEPS Hors série n¹ Décembre 2015}

La mise en œuvre de ce choix tactique suppose du dynamisme, de l'intensité et de la résolution. Par conséquent, la défense ne doit pas se borner simplement à des répliques données aux actions des adversaires, mais à des ripostes qui obligeront en permanence l'attaque adverse à se centrer sur la conservation du ballon et à ne pas agir en priorité pour la réalisation d'un point. Quand la tactique défensive réussit, l'attaque perd la balle.

Le dernier point concernant la réversibilité est en rapport avec les compétences motrices et leur exécution. En effet, la difficulté avec celles-ci, est qu'elles sont susceptibles de se dégrader avec la fatigue du stress ou une baisse d'attention. Cela se produit également avec un rapport de forces trop déséquilibré qui fait que les joueurs sont toujours en retard sur le jeu. Ce rapport de forces négatif fait basculer les joueurs dans un statut de «dominé »... avec ses conséquences sur les modélisations du jeu. Par exemple, la possession de la balle en attaque peut se réduire à sa simple mais problématique conservation quand l'adversaire est trop largement supérieur et que le principe de «l'égalité des chances à l'inégalité du résultat » ne peut plus être respecté (Gréhaigne, Mahut \& Marchal, 1998). Le joueur de sport collectif est aussi le résultat, à cet instant précis, d'une relation dialectique entre le poste qu'il occupe, ses qualités physiques, ses ressources motivationnelles, ses connaissances, etc. Cet état d'équilibre est souvent précaire et sa principale caractéristique est la réversibilité. Un joueur, en fonction d'un rapport de forces vécu défavorablement, peut revenir à des conduites, relevant d'une adaptation à l'affrontement, inférieures à son niveau théorique. Cette approche devrait conduire à proposer une autre conception des conduites communes à tous les joueurs. Cette conception plurielle prenant en compte comme fait premier et signifiant les différences entre les adaptations mises en œuvre, dans la même situation par des individus différents ou par le même individu en des moments différents.

En conclusion de ce paragraphe, la logique qui a présidé à la construction de cette analyse vise à tenir compte du rapport de forces dans un système d'opposition en situation de jeu collectif. Cet ensemble constitue une trame dynamique de transformation où la réversibilité souligne l'immédiateté du passage d'attaquant à défenseur, et met bien en évidence la notion de "situation à double effet" (Deleplace, 1979). II convient néanmoins de souligner que l'organisation de la réversibilité et / ou de la stabilité constitue un projet en soi à l'intérieur d'un projet de jeu plus général et qu'il nécessite, au cours du temps, la mise en place de règles de fonctionnement précises. 


\section{eJRIEPS Hors série n¹ Décembre 2015}

\section{Organisation et auto-organisation du jeu}

Le jeu n'est pas une chose amorphe que l'on devrait animer. Le jeu est création permanente du fait des rapports d'opposition qui apportent sans cesse fluctuation et évolution. Une de ses particularités réside dans sa capacité d'auto-organisation. Parfois la notion d'auto-organisation est associée à la notion d'émergence. C'est un phénomène dit émergent lorsqu'on ne peut pas prédire son apparition au sein du système dans lequel il peut survenir et dont il dépend fondamentalement. Pour nous, les configurations émergentes possèdent bien d'authentiques caractéristiques autonomes mais peuvent faire, parfois, l'objet de descriptions. Risque vs sécurité en relation avec avance ou retard sont alors des outils qui permettent de mieux apprécier la configuration momentanée du jeu en train d'évoluer.

6. 1.Différents niveaux d'action de jeu

Cette notion d'organisation impose, en particulier, dans l'examen des configurations du jeu, la description de niveaux d'organisation avec l'idée sous-jacente d'une complexité à étudier. Ainsi, dans une rencontre de football, nous avons identifié plusieurs systèmes emboîtés constituant autant de niveaux d'organisation en interaction (Gréhaigne, 1989, 1992 ; Gréhaigne, Bouthier \& David, 1997 ; Gréhaigne, Godbout \& Bouthier, 1999) produisant des configurations particulières. Concernant spécifiquement les effets des rapports d'opposition dans le déroulement d'une quelconque séquence de jeu, nous distinguerons trois niveaux différents d'affrontement (Gréhaigne, 1989). Tout d'abord, le niveau d'organisation « match » qui comprend l'effectif total des deux équipes. C'est le jeu de plein mouvement avec l'engagement de la totalité des deux équipes. Ensuite, le niveau d'organisation « affrontement partiel » qui oppose des sous-groupes pouvant aller jusqu'à la moitié de l'effectif de chaque équipe. Enfin, le niveau d'organisation « 1 contre 1 » qui représente un duel particulier à l'intérieur du niveau d'organisation « affrontement partiel ». Bien sûr, les effets s'engendrent les uns les autres et rejaillissent à la fois dans la simultanéité de l'instant et la succession des instants. L'analyse de la dynamique du jeu permet non seulement de percevoir les facteurs contribuant à réguler le jeu mais aussi d'appréhender différents niveaux de régulation et leur interdépendance. Elle conduit également à bien replacer l'activité du joueur et/ou du groupe dans un contexte plus global qui lui donne sa signification. En effet, l'ajustement à la réalité du moment est un processus qui se développe à deux niveaux de façon indissociable. 


\section{eJRIEPS Hors série n¹ Décembre 2015}

- Un processus interne qui est l'organisation ou la réorganisation des actions en vue de rétablir la cohérence des réponses du joueur ou du groupe de joueurs à la situation présente.

- Un processus externe qui intègre ces réponses à un ensemble plus vaste représenté par l'affrontement des deux équipes et où l'émergence d'une configuration inattendue est possible.

Dans une rencontre, chaque équipe est caractérisée par un taux d'entropie (désordre) qui traduit le degré d'organisation ou de désorganisation du groupe. Ce taux est révélateur du fonctionnement collectif et doit néanmoins rester dans des valeurs acceptables pour permettre l'affrontement. Trop d'ordre apparent chez les attaquants impliquerait en effet une tactique aisée de récupération et de contre pour l'adversaire, tandis que trop de désordre réel induirait des aléas difficilement surmontables par les partenaires. Pour réussir, cela pourrait renvoyer à des stratégies collectives (soit circulations de balle préétablies, soit combinaisons tactiques) à condition de jouer plus vite que l'adversaire afin de conserver un temps d'avance sur le replacement défensif.

6. 2. Le niveau d'organisation « équipe » et son réseau de compétences Un réseau de compétences est constitué par les relations entre les joueurs à l'intérieur d'une équipe. Ce sont les conduites et les comportements qui peuvent être identifiés chez les élèves en fonction d'un rapport de forces ou de leur statut dans l'équipe. L'ensemble de ces conduites varie suivant les joueurs, les moments, les facteurs extérieurs et les sports collectifs... En jeu, la notion de rôle (conduite qui exprime ce que le joueur pense avoir à faire en rapport avec la façon dont il vit le rapport de forces, le réseau de compétences dans l'équipe et comment il gère ses ressources dans ce système de contraintes) est essentielle à l'analyse de ce type de comportements. La fonction dans le groupe que le joueur choisit ou qui lui est assignée par l'enseignant ou par le groupe est un autre révélateur de sa position dans la dynamique de l'équipe. A l'interface de la logique du sujet, de la logique du groupe et de la logique interne du sport considéré, la place du joueur dans ce réseau de compétences constitue, souvent, un révélateur fiable des rapports réciproques entre ce joueur et l'équipe. Parfois, cela peut prendre la forme d'un rapport de forces entre deux partenaires.

Dans chaque équipe s'instaurent, donc, une distribution des tâches voulues par quelquesuns, des conflits ou une répartition tacite des rôles et des fonctions générant ainsi un réseau de compétences. Pour évoluer, cette répartition des tâches demande du temps, l'établissement d'un bilan pour chaque joueur en vue d'optimiser les apports de tous au 


\section{eJRIEPS Hors série n¹ Décembre 2015}

collectif, tout en faisant que la collectivité permette à tous de s'améliorer et de s'exprimer dans tous les sens du terme.

6. 3. Le niveau d'organisation « joueur ».

Un joueur de sport collectif constitue un système complexe où de nombreux éléments en interaction composent différents niveaux d'organisation avec des états d'équilibre et de déséquilibre variés. Cet ensemble dépend, tout d'abord, de la façon dont le joueur vit le rapport de forces, l'opposition avec les adversaires et le rapport de compétence à l'intérieur de l'équipe.

Pour des joueurs, une même adaptation à un affrontement donné dépendra de la combinatoire de différents facteurs donc de la hiérarchie existant entre les perceptions, les compétences motrices, les règles de l'organisation du jeu et les règles d'action. Apprécier un joueur de sport collectif, c'est, donc, toujours le juger dans une organisation que constitue l'équipe. Les réponses apportées sont aussi dans les caractéristiques de la situation, les contraintes et les opportunités telles que la personne les perçoit. Prendre au sérieux le comportement de quelqu'un, c'est utiliser ce comportement pour découvrir une situation, faire du comportement un symptôme de la situation plutôt qu'un symptôme d'une personne. Cela permet d'utiliser l'observation des comportements pour remonter éventuellement à la structure profonde, aux règles du jeu social, aux arrangements par lesquels les gens vont structurer leur coopération dans un ensemble. Le modèle de fonctionnement, de développement et d'évaluation du joueur de sport collectif qui vient d'être esquissé met l'accent sur la pluralité des processus et la nature des relations qu'ils entretiennent dans le fonctionnement du joueur. Cette vision semble universelle dans la mesure où le répertoire des processus est le même pour tous les sujets. Elle est différentielle dans la mesure où la hiérarchie entre les éléments du système de variables est changeante selon l'affrontement, les équipes et / ou selon les individus. La figure 2 propose un exemple d'un système de variable pour analyser une configuration momentanée du jeu. 


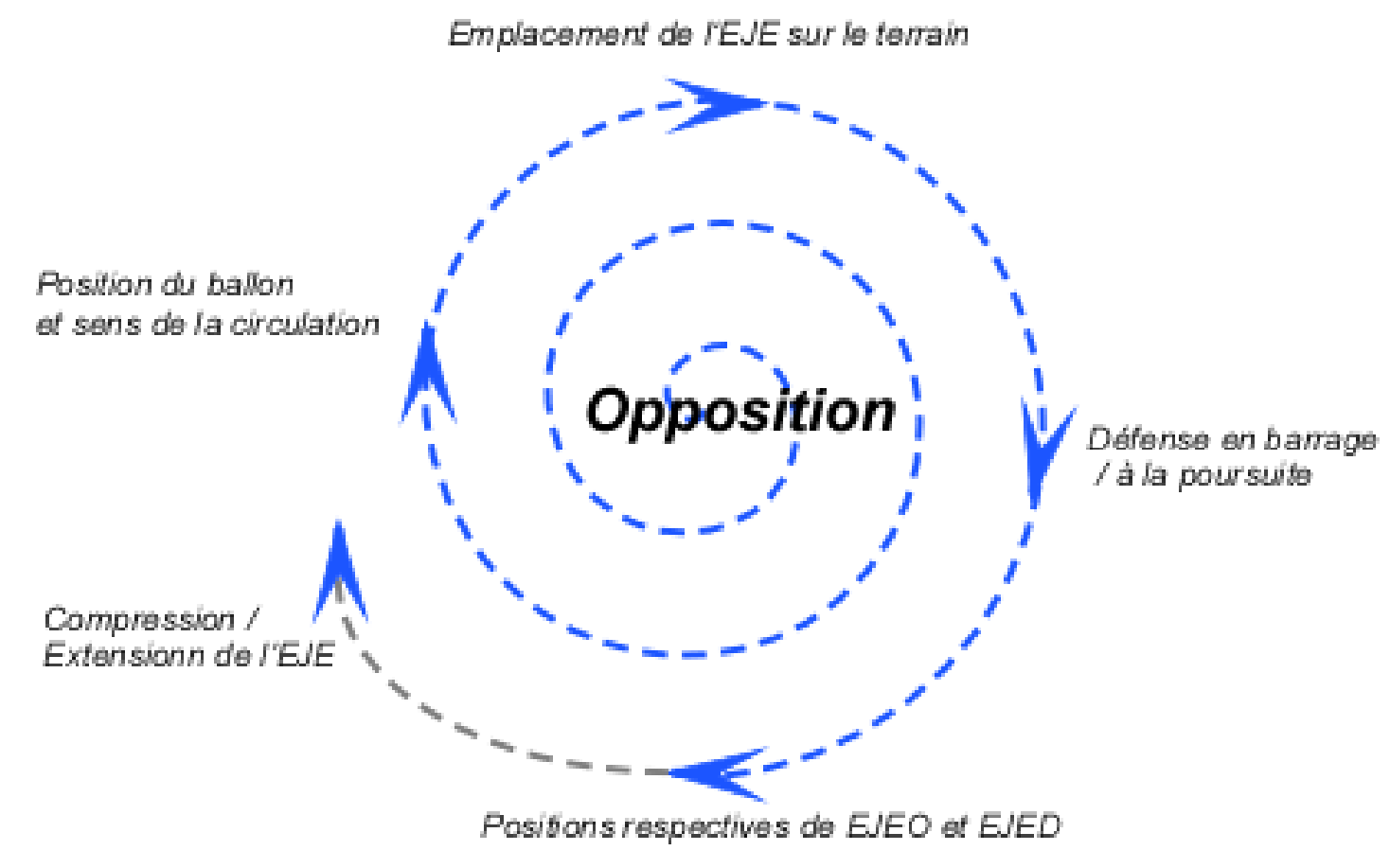

Figure 2. Critères d'analyse d'une configuration momentanée du jeu.

Ainsi, l'évolution de la dynamique du jeu ne peut alors se modéliser qu'en concevant une évolution discontinue dans le temps. II nous semble qu'une base de données constituée par la mise en relation de cinq critères 1) emplacement sur le terrain et volume de l'espace de jeu effectif (I'EJE), 2) la position et la circulation du ballon, 3) les positions respectives de EJEO et EJED, 4) la défense en barrage ou à la poursuite et enfin, 5) l'extension ou la compression de l'EJE, permet d'obtenir une représentation immédiate et assez exacte du rapport d'opposition (Figure 2).

\section{Conclusion}

L'objectif de ce travail était d'exposer quelques concepts utiles lors de la mise en place d'un enseignement des sports collectifs basé sur le jeu. Cette approche permet de se démarquer d'une didactique très analytique, centrée sur le geste, pour aller vers une approche plus globale basée sur une approche tactique des jeux.

L'ensemble de cette réflexion constitue une trame qui est à enrichir, à discuter et à faire évoluer en liaison avec la recherche sur la didactique de l'EPS afin que les contenus proposés aux élèves soient de plus en plus pertinents. 


\section{eJRIEPS Hors série n¹ Décembre 2015}

\section{Bibliographie}

Brackenridge, C. (1979). Games : Classification and analysis. Conférence présentée aux Kirkless Teachers, le 02 mai 1979.

Caillé, A. (Ed.) (2008). Identités de l'individu contemporain. Paris : Textuel / La Discorde.

Deleplace, R. (1979) Rugby de mouvement - Rugby total. Paris, Éducation Physique et Sports.

Deleplace, R. (1983). La recherche sur la spécialisation sportive, l'entraînement, la performance. In Actes du colloque la recherche en STAPS (pp. 93-151). Nice. 19-20 Septembre 1983.

Deleplace, R. (1994). La notion de matrice d'action pour les actions motrices complexes. In D. Bouthier \& J. Griffet (Eds.) Représentation et action en activité physique et sportive (pp. 25-42). Université de Paris-Orsay.

Eloi, S. \& Uhlrich, G. (2001). Contribution à la caractérisation des sports collectifs : les exemples du volleyball et du rugby. Revue STAPS, 56, 109-126.

Eloi, S., \& Uhlrich, G. (2011). La démarche technologique en STAPS : analyse conceptuelle et mise en perspective pour les sports collectifs. eJRIEPS, 23, 20-45.

Gréhaigne, J.-F. (1989). Football de mouvement. Vers une approche systémique du jeu. Thèse de Doctorat (non publiée). Dijon, France : Université de Bourgogne.

Gréhaigne, J.-F. (1992). L'organisation du jeu en football. Paris : ACTIO.

Gréhaigne, J.-F. (1992a). La notion d'opposition au cœur de la didactique des sports collectifs. In A. Menaut (Ed.) Méthodologie et pédagogie des sports collectifs (pp. 77-89). Université Victor Segalen Bordeaux 2.

Gréhaigne, J.-F. (Ed.) (2014). L'intelligence tactique. Des perceptions aux décisions tactiques dans les sports collectifs. Besançon : Presses de l'Université de Franche-Comté.

Gréhaigne, J.-F., Bouthier, D., \& David, B. (1997). Dynamic systems analysis of the opponent relationships in the collective actions in soccer. Journal of Sports Sciences, 15, 137-149.

Gréhaigne, J.F., Godbout, P., \& Bouthier, D. (1999). The foundations of tactics and strategy in team sports. Journal of Teaching in Physical Education, 18, 159-174.

Gréhaigne, J.F., Mahut, N., \& Marchal, D. (1998). Les nomogrammes en EPS : mode d'emploi et approche critique. In C. Amade-Escot, J.P. Barrué, J.C. Bos, F. Dufor, M. Dugrand, \& A. Terrisse (Eds.), Recherches en EPS, bilan et perspectives (pp. 329-340). Paris : Revue EPS.

Jeu, B. (1977). Le sport, l'émotion, l'espace. Paris : Vigot.

Jullien, F. (1996). Traité de l'efficacité. Paris : Grasset.

Lévi-Strauss, C. (1955). Tristes tropiques. Paris : Plon.

Malho, F. (1969). L'acte tactique en jeu. Paris : Dunod.

Marle, P., Pasteur, R., \& Voland, C. (1996). Enseigner en sport collectif avec le football. Paris : AEEPS.

Menaut, A. (1982). Contribution à une approche théorique des jeux sportifs collectifs. Thèse de doctorat d'état (non publiée), Université de Bordeaux 2.

Mérand, R. (1977). L'éducateur face à la haute performance. Paris : Editions Sport et plein air.

Piaget, J. (1974). Réussir et comprendre. Paris : PUF.

Villepreux, P. (1987). Rugby de mouvement et disponibilité du joueur. Mémoire INSEP, Paris. 